\title{
Thermoelectric effects in a rectangular Aharonov-Bohm geometry
}

\author{
A. J. Pye, ${ }^{1}$ D. A. Faux, ${ }^{1}$ and M. J. Kearney ${ }^{1}$ \\ Faculty of Engineering and Physical Sciences, University of Surrey, \\ Guildford, Surrey, GU2 $7 X H$, United Kingdom.
}

The thermoelectric transport properties of a rectangular Aharonov-Bohm ring at low temperature are investigated using a theoretical approach based on Green's functions. The oscillations in the transmission coefficient as the field is varied can be used to tune the thermoelectric response of the ring. Large magnitude thermopowers are obtainable which, in conjunction with low conductance, can result in a high thermoelectric figure of merit. The effects of single site impurities and more general Anderson disorder are considered explicitly in the context of evaluating their effect on the Fano-type resonances in the transmission coefficient. Importantly, it is shown that even for moderate levels of disorder the thermoelectric figure of merit can remain significant, increasing the appeal of such structures from the perspective of specialist thermoelectric applications. 


\section{INTRODUCTION}

Recent developments have seen growing interest in the realization of small scale electron waveguide structures for specialist thermoelectric applications, based on enhanced performance through tuning electronic transmission together with reducing phonon contributions ( see $^{1,2}$ and references therein). Such structures include single and multiple bend configurations, leading to the natural question as to the level of performance one might expect from ring geometries. Small ring structures have been of interest in the field of quantum transport since Aharonov and Bohm first predicted in 1959 that magnetic flux through the ring would affect the behavior of electrons passing through the structure even if the electrons do not experience the flux directly ${ }^{3}$. However, at that time the manufacturing techniques required to fabricate such a ring did not exist. Approximately twenty years later, quantum electronic transport saw a surge in popularity as it became possible to make structures small enough to demonstrate quantum effects. This led to renewed interest in both the theoretical ${ }^{4}$ and experimental study of rings, with Aharonov and Bohm's initial predictions eventually being confirmed experimentally for circular gold rings ${ }^{5}$ and later for a square ring made of antimony ${ }^{6}$.

The thermoelectric properties of small rings have been studied by a number of authors. It is known that the Aharonov-Bohm behavior provides a means of tuning the thermopower and hence thermoelectric efficiency via the flux through the ring ${ }^{7}$. Studies of Aharonov-Bohm interferometers containing single ${ }^{8-11}$ and double ${ }^{12-15}$ quantum dots have been carried out leading to a number of interesting findings, including the prediction of Fano-type resonances in the transmission coefficient and therefore the conductance. These can lead to respectable thermoelectric performance ${ }^{15}$ which can be enhanced by tuning of the flux. Indeed, it has been argued that a thermoelectric figure of merit approaching 4 could be attainable for such devices at room temperature ${ }^{11}$. More recent studies have focussed further on issues relating to the exploitation of these Fano-type resonances ${ }^{16}$, as well as investigations into decoherence $^{17-20}$.

In this paper we focus on the study of rectangular rings, in relation to which some interesting results have already been found. For example, $\mathrm{Wu}$ and co-workers ${ }^{21}$ applied a spin-dependent superlattice-like potential to each arm of such a ring allowing the structure 
to function as a highly-effective spin filter to generate spin-polarized current. From an applications perspective, a rectangular geometry may also confer additional advantages over, say, a circular geometry in terms of ease of manufacture, as well as possibly defining sharper resonances (and hence stronger thermoelectric response) due to the enhanced scattering potential presented by the abrupt angular bends.

Here we explore theoretically the thermoelectric aspects of the transport and the effects of a magnetic field at low temperatures. We ignore the role played by phonons, recognizing that at sufficiently low temperatures their contribution will be relatively insignificant and could, if desired, be considered as a perturbation. Of course, a complete understanding of the thermoelectric properties of such structures at more elevated temperatures requires an understanding of how phonons contribute to thermal conductivity, which plays a role in determining the overall thermoelectric figure of merit, and how this phonon contribution is modified by geometric constraints (a recent review may be found $\mathrm{in}^{22}$ ). We do, however, consider the consequences of structural imperfections by modeling the effects of single impurities and random disorder inside the ring. This is an important consideration recognising the inevitable challenges which arise when fabricating devices on such a small scale.

The paper is organized as follows. In Sec. II an overview of the theory is presented, paying attention to the seldom discussed matter of how one can best allow for the effects of the magnetic field within the attached leads. Results are presented in Sec. III for the transmission coefficient, the electrical conductance and the thermopower, and the physics underlying the behavior exhibited by the rectangular ring is discussed both in the absence and presence of disorder. Finally, in Sec. IV a summary of the key findings is presented and conclusions are drawn.

\section{THEORY}

The theory we use is based on the Landauer-Büttiker picture of quantum transport ${ }^{23-26}$; see also $^{2}$ for further details. The system consists of a device region attached to two semiinfinite leads, each terminating in a reservoir. The reservoirs are considered to be large enough that the addition or removal of a carrier has a negligible effect on their internal 
state so that each reservoir acts as a source and sink for carriers in the lead to which it is attached with all equilibration occurring in the reservoir. The states in each lead are therefore maintained at a local equilibrium temperature and chemical potential determined by the reservoir.

A Green's function method ${ }^{27}$ is used to evaluate the transport properties of the system shown in Fig. 1. It is assumed that the $z$-direction decouples from the $x$ - and $y$-directions so that the system may be assumed to be two-dimensional. The physics of the device is described by the time-independent Schrödinger equation for electrons of charge $-|e|$ and effective mass $m^{*}$,

$$
\left[\frac{(-i \hbar \boldsymbol{\nabla}+|e| \boldsymbol{A})^{2}}{2 m^{*}}+U(x, y)\right] \psi(x, y)=E \psi(x, y)
$$

where $\boldsymbol{A}$ is the vector potential which defines the magnetic field via the relation $\boldsymbol{B}=\boldsymbol{\nabla} \times \boldsymbol{A}$. The potential $U(x, y)$ serves two purposes. Firstly, the geometry of the device is defined by setting $U(x, y)$ to have a large value in the areas indicated by hatching in Fig. 1 which creates exclusion zones from the perspective of the electrons. Secondly, it allows us to include the effects of impurities and general disorder as described later.

For the purpose of numerical computations we perform a discretization of real space using a grid with spacings of $a_{0}$ in both the $x$ - and $y$-directions. The device region contains $N=N_{x} \times N_{y}$ points, with the $n^{\text {th }}$ point having a position denoted as $\boldsymbol{r}_{n}$. We choose to write the vector potential as

$$
\boldsymbol{A}=-B\left(y-L_{y} / 2\right) \hat{\boldsymbol{x}}
$$

where $B$ is constant so that the magnetic field is given by $\boldsymbol{B}=B \hat{\boldsymbol{z}}$. This satisfies the Coulomb gauge condition $\boldsymbol{\nabla} \cdot \boldsymbol{A}=0$ which is particularly natural for our geometry and discretization procedure.

The expression in the square brackets in (1) is the Hamiltonian which, when discretized, gives an $N \times N$ matrix with elements ${ }^{28}$

$$
[\hat{H}]_{n m}= \begin{cases}4 t_{0}+U_{n}, & n=m \text { (on-site energy) } \\ -t_{0} \phi_{n}, & n \text { and } m x \text {-adjacent } \\ -t_{0}, & n \text { and } m y \text {-adjacent } \\ 0, & \text { otherwise }\end{cases}
$$




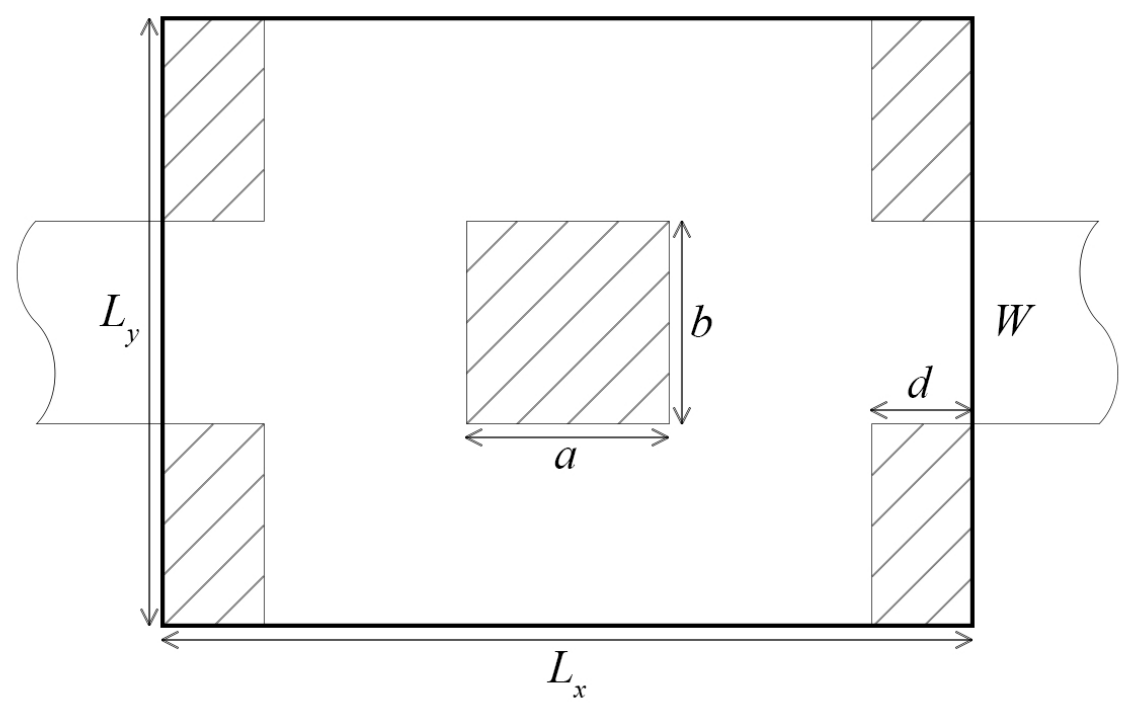

FIG. 1: The rectangular ring studied. The area of dimensions $L_{x} \times L_{y}$ marked by the bold line is considered as the device region. Hatched areas represent exclusion zones where a large potential is applied so that a central forbidden region of dimensions $a \times b$ is formed. A length $d$ of each lead is included in the device region. Leads of width $W$ are attached to the left and right sides of the device region.

where the energy $t_{0}=\hbar^{2} / 2 m^{*} a_{0}^{2}$, and a subscript $n$ denotes the value of that function taken at position $\boldsymbol{r}_{n}$ on the grid. The factor $\phi_{n}$ is the so-called Peierls phase factor ${ }^{29}$,

$$
\phi_{n}= \begin{cases}e^{i|e| a_{0}\left[A_{x}\right]_{n} / \hbar}, & m \text { to the right of } n, \\ e^{-i|e| a_{0}\left[A_{x}\right]_{n} / \hbar}, & m \text { to the left of } n,\end{cases}
$$

which captures the phase "picked up" by an electron traveling between nearest-neighbor points in the $x$-direction. The fundamental quantity required for the transport calculations is the (discretized) retarded Green's function for the device region,

$$
G=a_{0}^{-2}[(E+i \eta) I-\hat{H}-\Sigma]^{-1}
$$

where $\eta$ is an infinitesimal positive quantity used to enforce the boundary conditions and $\Sigma$ is a self-energy matrix representing the effect of the semi-infinite leads on the device.

Before the expression for the self-energy is presented, we consider the question as to how to treat the magnetic field in the leads. In the Landauer-Büttiker picture, the leads serve 
the purpose of supplying electrons to the device in pre-prepared states and returning them to the reservoirs for thermalization. Whilst the leads themselves are not fundamental to determining the physics of the device, the states in the leads in the vicinity of the device are important as regards determining the self-energy appearing in Eq. (5) and must be described accurately. Therefore, in what follows, a length $d$ of each lead is included in the device region (see Fig. 1) in which the magnetic field is accounted for in full by the Peierls factors entering the Hamiltonian given in Eq. (3). Provided $d$ is large enough, the effect of the magnetic field in the parts of the leads beyond the device region becomes insignificant and can be neglected. In principle we must have $d \rightarrow \infty$ to perfectly recreate the states of a semi-infinite lead in the presence of a magnetic field. However, our numerical testing has shown that even relatively small values of $d$ are sufficient to provide a good approximation. It is therefore assumed that the vector potential in the parts of the leads outside the device region is zero so that the zero-field expressions for quantities involving the leads may be used. Even if the vector potential is non-zero at the boundary between the leads and the device region, there is no spurious contribution to the magnetic field by setting it to zero in the leads since this constitutes a change in the $x$-component of the vector potential in the $x$-direction, i.e. $\partial A_{x} / \partial x$, which makes no contribution to $\boldsymbol{B}$. This is guaranteed because of our choice of gauge.

The two leads are labelled by $p(p=1,2)$ and are assumed to have a square-well crosssection of width $W$ centered at $y_{p}=0$. Since the magnetic field in the leads beyond the device region is neglected, the transverse mode with index $\alpha$ has the wavefunction

$$
u_{\alpha}\left(y_{p}\right)=\sqrt{\frac{2}{W}} \sin \left(\frac{\alpha \pi\left(y_{p}+\frac{W}{2}\right)}{W}\right)
$$

and energy

$$
\epsilon_{\alpha}=\frac{\hbar^{2} \pi^{2} \alpha^{2}}{2 m^{*} W^{2}}
$$

Following the discussion $\mathrm{in}^{27}$ and assuming that there is no vector potential in the leads, the self-energy matrix elements for lead $p$ are found to be

$$
\left[\Sigma_{p}\right]_{n m}= \begin{cases}-t_{0} a_{0} \sum_{\alpha} u_{\alpha}\left(y_{p_{n}}\right) u_{\alpha}\left(y_{p_{m}}\right) e^{i k_{\alpha} a_{0}}, & \boldsymbol{r}_{m} \text { and } \boldsymbol{r}_{n} \text { adjacent to lead } p \\ 0, & \text { otherwise }\end{cases}
$$

where $u_{\alpha}\left(y_{p_{n}}\right)$ is the value of $u_{\alpha}$ at point $y_{p_{n}}$ and $k_{\alpha}=\sqrt{2 m^{*}\left(E-\epsilon_{\alpha}\right) / \hbar^{2}}$ is the longitudinal wavenumber for mode $\alpha$. The sum over the transverse modes is limited to those for which 
$\epsilon_{\alpha}<E$ so that only energetically-available propagating states are included. The total selfenergy $\Sigma$ appearing in (5) is $\Sigma=\Sigma_{1}+\Sigma_{2}$.

The individual (lead specific) self-energy matrices are used to find the broadening matrix for lead $p$,

$$
\Gamma_{p}=\Sigma_{p}-\Sigma_{p}^{\dagger}
$$

where the dagger denotes the conjugate transpose. The Green's function and broadening matrices can be used to define and calculate a local density of states at a point $\boldsymbol{r}_{n}$ as

$$
D_{n}=a_{0}^{2} \frac{\left[G \Gamma G^{\dagger}\right]_{n n}}{2 \pi}
$$

where $\Gamma=\Gamma_{1}+\Gamma_{2}$. Alternatively we can replace $\Gamma$ in (10) with $\Gamma_{p}$ to obtain a measure of the "contribution" of lead $p$ to the total density of states.

The key quantities in determining the transport characteristics of the rectangular ring are the transmission coefficients $t_{q p}$ which give the relative probability of an electron incident on the device from lead $p$ to exit via lead $q$. Using the Fisher-Lee relation ${ }^{30}$ and again following the discussion in $^{2}$ and $^{27}$, these are

$$
t_{q p}=\delta_{q p}\left\{M_{p}+2 a_{0}^{2} \operatorname{Tr}\left[\Gamma_{p} \operatorname{Im}(G)\right]\right\}+a_{0}^{4} \operatorname{Tr}\left[\Gamma_{q} G^{\dagger} \Gamma_{p} G\right]
$$

where $M_{p}=\sum_{\alpha} 1$ is the number of occupied modes in lead $p$, Tr is the trace and $\operatorname{Im}(G)$ denotes the imaginary part of $G$. The first term in Eq. (11) only contributes to reflection probabilities and is not usually seen in the literature since these can be obtained using only the transmission coefficients and the conservation of probability. However, the term is retained here for the sake of completeness.

The electrical conductance and thermopower can be calculated once the transmission coefficients are known. The chemical potential $\mu_{p}$ and temperature $T_{p}$ of the reservoir to which the lead is attached are defined for each lead. It is assumed that the potential and temperature differences between the leads are small so that $\mu_{1} \approx \mu_{2} \approx \mu$ and $T_{1} \approx T_{2} \approx T$. In this regime the linear conductance $G$ and thermopower $S$ are given by ${ }^{31,32}$

$$
\begin{aligned}
G & =\frac{2 e^{2}}{h} \int_{\epsilon_{1}}^{\infty}\left(-\frac{\partial f_{0}}{\partial E}\right) t(E) \mathrm{d} E \\
S & =-\frac{L}{G}
\end{aligned}
$$


where $f_{0}$ is the Fermi function at energy $E$ and thermal energy $k_{B} T, t(E)=t_{12}=t_{21}$, and $L$ is given by

$$
L=-\frac{2|e|}{h T} \int_{\epsilon_{1}}^{\infty}\left(-\frac{\partial f_{0}}{\partial E}\right)(E-\mu) t(E) \mathrm{d} E .
$$

Each of these quantities is evaluated at low temperature and we assume, as discussed in the Introduction, that any effects due to phonons are negligible. Results for the conductance are presented in units of $G_{0}=e^{2} / h$, the quantum of electrical conductance per spin. Finally, to measure the thermoelectric efficiency of the rectangular ring, the thermoelectric figure of merit $Z T$ is determined from ${ }^{33}$

$$
Z T=\frac{G S^{2} T}{\kappa}=\frac{L^{2} T}{G \kappa}
$$

where $\kappa$ is the thermal conductance,

$$
\kappa=-K\left(1+\frac{S^{2} G T}{K}\right)=-K-\frac{L^{2} T}{G}
$$

and

$$
K=-\frac{2}{h T} \int_{\epsilon_{1}}^{\infty}\left(-\frac{\partial f_{0}}{\partial E}\right)(E-\mu)^{2} t(E) \mathrm{d} E .
$$

In situations where the transmission coefficient is a slowly-varying function of energy, the behavior of $\kappa$ at low temperature is similar to $G$ multiplied by a scaling factor $G_{\text {th }} / G_{0}$, where $G_{\text {th }}=k_{B}^{2} \pi^{2} T / 3 h$ is the quantum of thermal conductance per spin. However, when the transmission coefficient is a rapidly varying function of energy more complex behavior is seen, which can lead to a significant enhancement of the overall thermoelectric figure of merit ${ }^{2}$. This is why Fano-type resonances in the transmission coefficient can play a prominent role in the thermoelectric behavior of the device ${ }^{16}$.

\section{RESULTS AND DISCUSSION}

Transmission plots for a range of values of the magnetic field are presented in Fig. 2. The representative parameters used are $W=a=b=20 \mathrm{~nm}, d=16 \mathrm{~nm}$ and an effective mass $m^{*}=0.067 m_{0}$ appropriate to GaAs. Changing these parameters has some effect on the

details of the transmission coefficient, but the general features observed are unchanged. For this reason, these parameters are used for all of the results presented. 


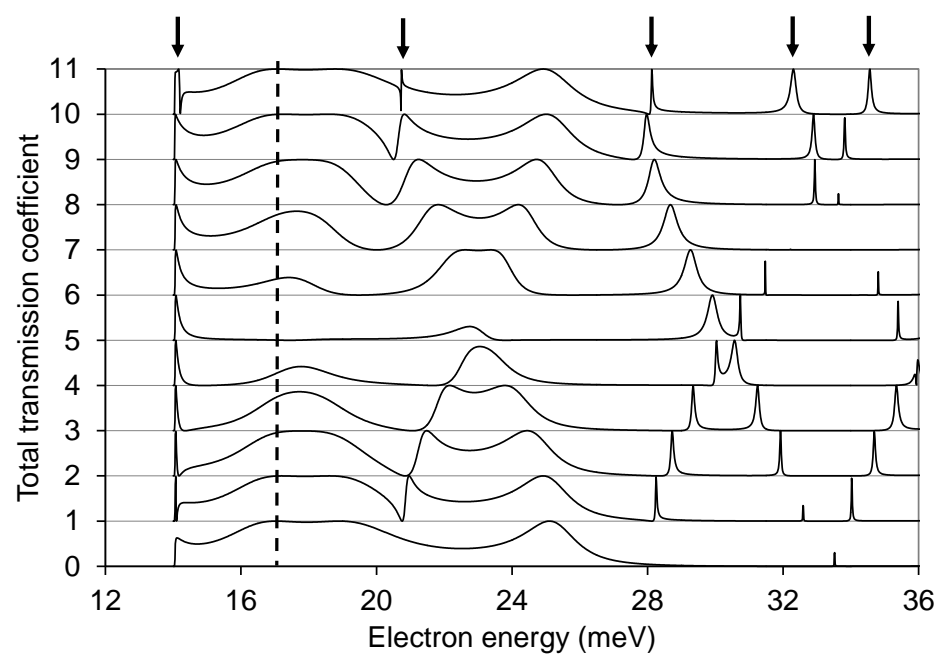

FIG. 2: Transmission plots of a rectangular ring for different magnetic field strengths. Curves for different values of the magnetic field are offset for clarity: from bottom to top, the values of $B$ range from 0 to $2.5 \mathrm{~T}$ in $0.25 \mathrm{~T}$ increments. The Fano-like resonances at $2.5 \mathrm{~T}$ are indicated by arrows. The dashed line at $17 \mathrm{meV}$ shows the scan for Fig. 3.

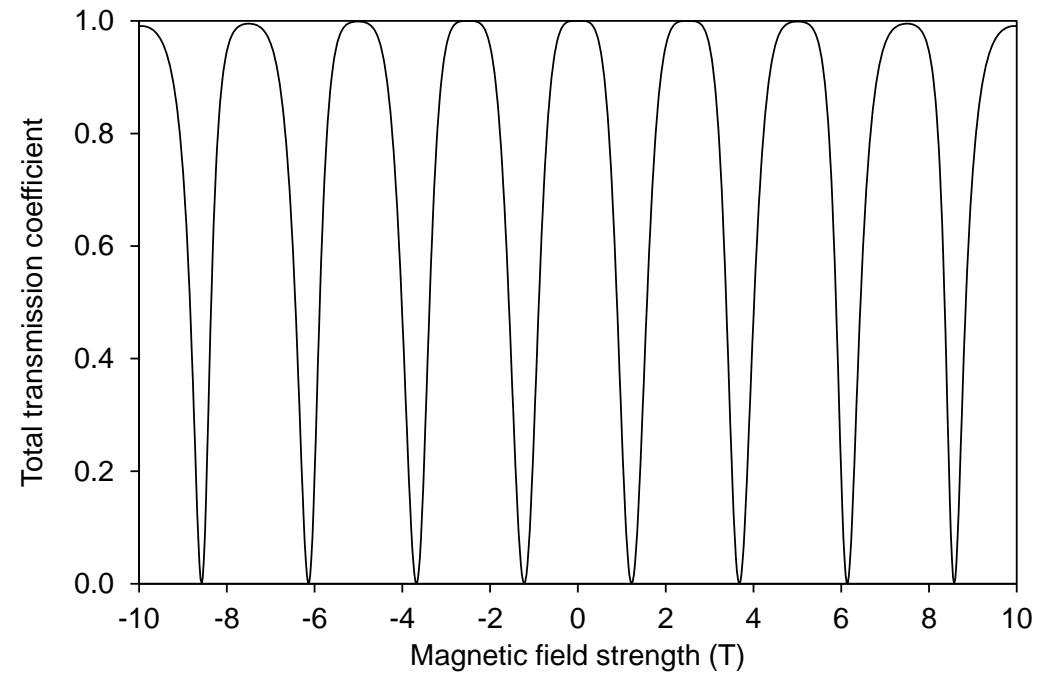

FIG. 3: Transmission coefficient for the rectangular ring at a constant energy $E \approx 17 \mathrm{meV}$. The features for the energy range $0-2.5 \mathrm{~T}$ correspond to the scan line in Fig. 2.

The results in Fig. 2 show that at zero field the transmission behavior is qualitatively similar to that of electron waveguides containing right-angle bends ${ }^{34}$ with a sharp cut-off 
region (below $14 \mathrm{meV}$ ) where transmission is not allowed followed by a peak structure and eventually another cut-off region above about $30 \mathrm{meV}$ which is punctuated by sharp resonances. As the magnetic field is increased from $0 \mathrm{~T}$, the transmission peaks shift in energy and begin to decrease in magnitude. When $B=1.25 \mathrm{~T}$, most of the peaks have disappeared completely leaving the transmission coefficient close to zero for the whole energy range shown. When $B$ is increased further, the reverse progression is seen until, at $2.5 \mathrm{~T}$, the results are similar in some (but not all) respects to those obtained at zero field. This is a consequence of the Aharonov-Bohm effect ${ }^{3}$, where the electrons traversing the device pick up a phase shift due to the vector potential. The phase shift is different from a pathtrajectory perspective depending on which arm of the ring the path takes, resulting in interference between the two paths through the structure. Theory predicts that the period of the resulting oscillation is given by $B_{p}=h /|e| C$, where $C$ is the area enclosed by the two paths. If it is assumed that the dominant paths follow the transverse center of the waveguides, $C \approx(40 \mathrm{~nm})^{2}$ for the parameters chosen so that $B_{p} \approx 2.6 \mathrm{~T}$, which is in good agreement with the results shown in Fig. 2. Similar behavior has been observed experimentally in the magnetoresistance of a square ing $^{6}$.

In Fig. 3 the transmission coefficient at a constant energy corresponding to the first peak seen in the zero field curve of Fig. 2 is presented as a function of the magnetic field over a range of approximately $-4 B_{p}<B<4 B_{p}$. The Aharonov-Bohm oscillations are clearly seen. In this particular case the periodic behavior is present over a number of cycles and the period remains largely unchanged throughout this range.

The linear-response transport properties of the rectangular ring are now considered. At low temperature, the conductance is expected to be a slightly smoothed version of the transmission coefficient whilst the thermopower is predicted to be roughly proportional to the gradient of the transmission curve ${ }^{35,36}$. Figure 4 illustrates these behaviors and also clearly shows that the thermoelectric response of the ring is significantly altered by the magnetic field. The resonances in the transmission coefficient give rise to peaks in the thermopower, which in conjunction with low electronic thermal conductance (not shown, but determined by Eq. (16) and Eq. (17) can lead to extremely large figures of merit (determined by Eq. (15)), peaking at values of approximately 30,10 and 5 for $B=0,1.25$ and $2.5 \mathrm{~T}$ respectively. This is shown in Fig. 5, where the figure of merit is plotted explicitly as a function of 

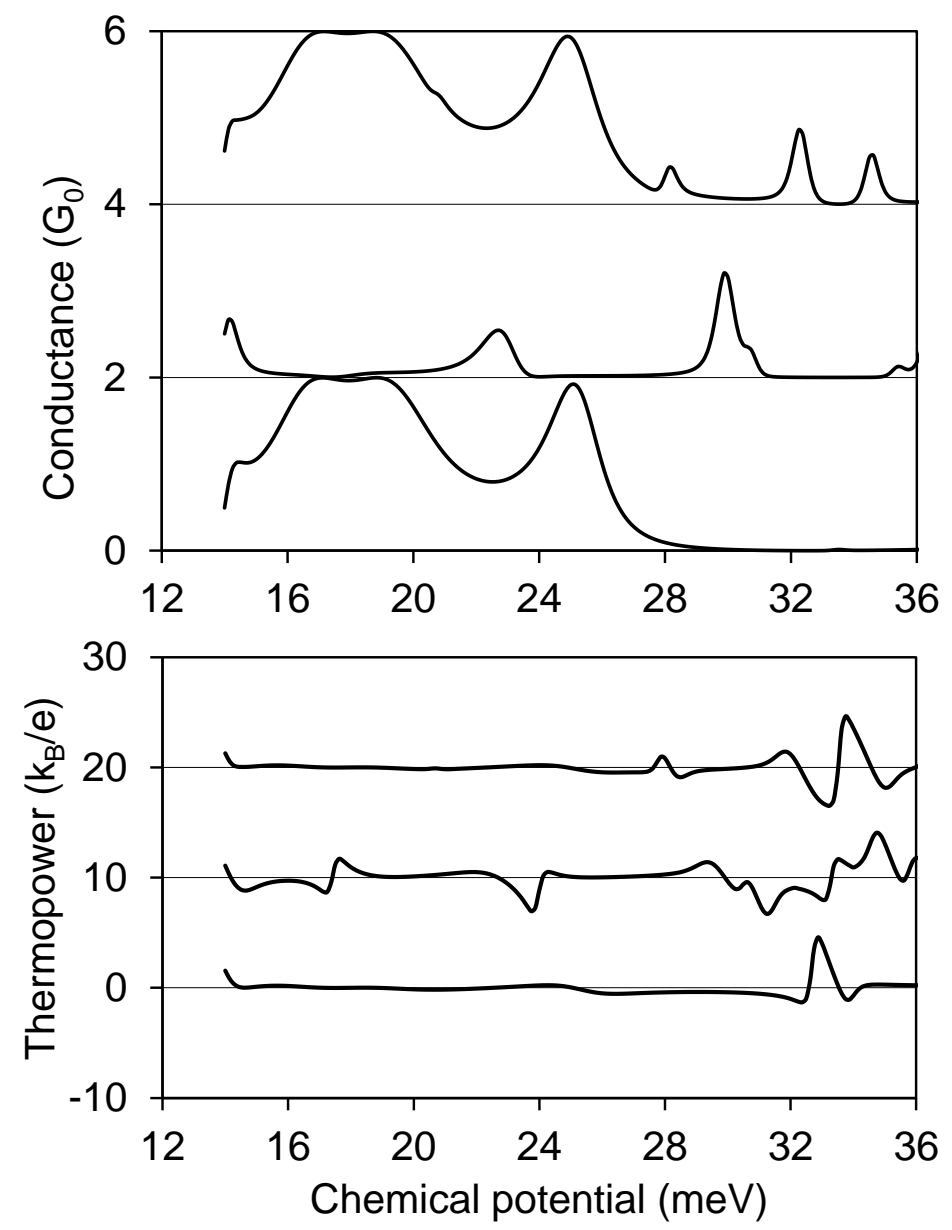

FIG. 4: Linear response conductance (top) and thermopower (bottom) for the rectangular ring with thermal energy $k_{B} T=0.125 \mathrm{meV}(T \approx 1.4 \mathrm{~K})$. Curves for different values of the magnetic field are offset for clarity, with $B=0,1.25$, and 2.5 T from bottom to top.

chemical potential for three different values of the magnetic field. For certain values of the chemical potential the figure of merit can significantly exceed unity, and the magnetic field offers the opportunity to tune the value for a given chemical potential. As mentioned in the Introduction, the presence of phonons will enhance the thermal conductance and therefore reduce these figures of merit, which should therefore be viewed as indicative. It is interesting and important to note that the transport coefficients (and hence the figure of merit) do not 


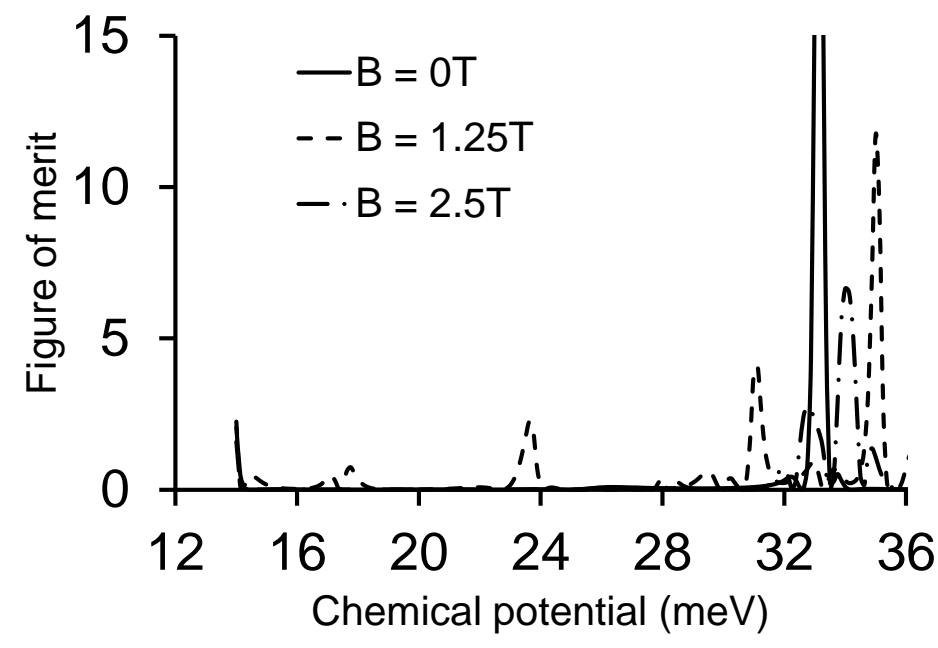

FIG. 5: Figure of merit for the rectangular ring with $B=0,1.25$, and 2.5T.

show full periodicity with magnetic field.
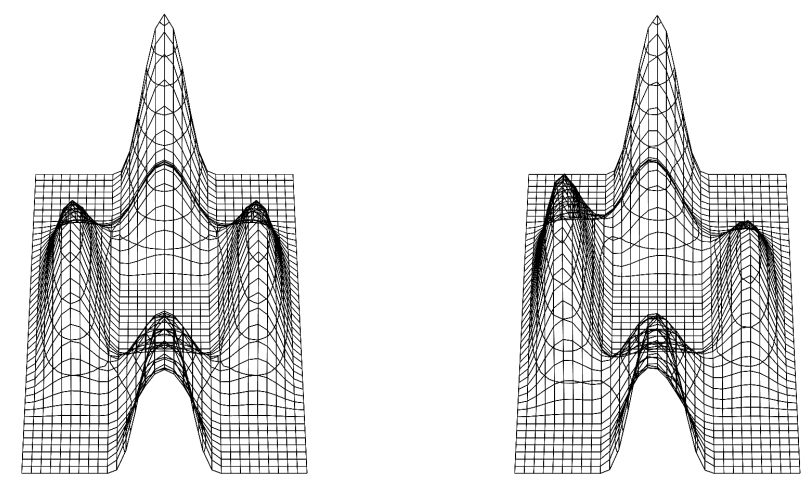

FIG. 6: Contribution to the effective local density of states in the rectangular ring coupled to the left lead viewed from above the left lead. The magnetic field is $B=0 \mathrm{~T}$ (left) and $B=2.5 \mathrm{~T}$ (right) and the energy in both cases is $E \approx 17 \mathrm{meV}$, corresponding to the first peak in the zero field transmission results.

An interesting aspect of the transmission coefficients presented in Fig. 2 is that, whilst the curves for $B=0 \mathrm{~T}$ and $B=2.5 \mathrm{~T} \approx B_{p}$ are similar, the $2.5 \mathrm{~T}$ results show clear 
Fano-like resonances at energies of approximately 14, 20, 28, 32 and $34 \mathrm{meV}$. Other authors have reported these for Aharonov-Bohm interferometers consisting of two coupled quantum $\operatorname{dots}^{12,13}$, finding that the resonances vanish if the coupling between the two dots is perfectly in series or in parallel. From this evidence they concluded that the resonances are the result of asymmetry between the possible paths an electron can take through the device. Our results confirm this conclusion. Although the geometry is perfectly symmetric, from a classical perspective the Lorentz force experienced by the electrons in the presence of a magnetic field means that they will couple preferentially to either the upper or lower arm of the ring depending on which lead they are incident from. This conclusion is supported by Fig. 6 which shows that the effective local density of states coupled to the left lead (see Eq. (10)) is larger in the upper arm than in the lower arm when a magnetic field is applied, but the two are identical at zero field.

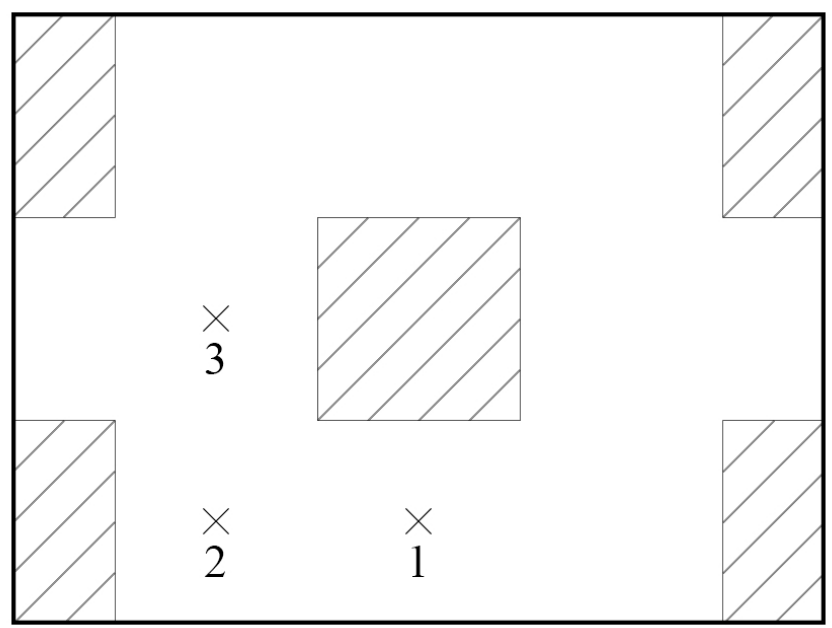

FIG. 7: Locations at which the single impurity is positioned in the ring. Placing the impurity at site 1 results in a break of symmetry in the $y$-direction only, whilst site 3 breaks symmetry only in the $x$-direction. Site 2 breaks symmetry in both the $x$ - and $y$-directions.

The discretized approach adopted for the present calculations makes it straightforward to probe this phenomenon further, and to investigate the important role of disorder (or symmetry breaking) in general, by placing a single point impurity at a number of different locations within the ring. This is achieved by adding an energy $V_{\text {imp }}$ to the potential at the grid point at which the impurity is situated. We measure $V_{\text {imp }}$ in terms of $t_{0}$ with $a_{0}=2 \mathrm{~nm}$ 

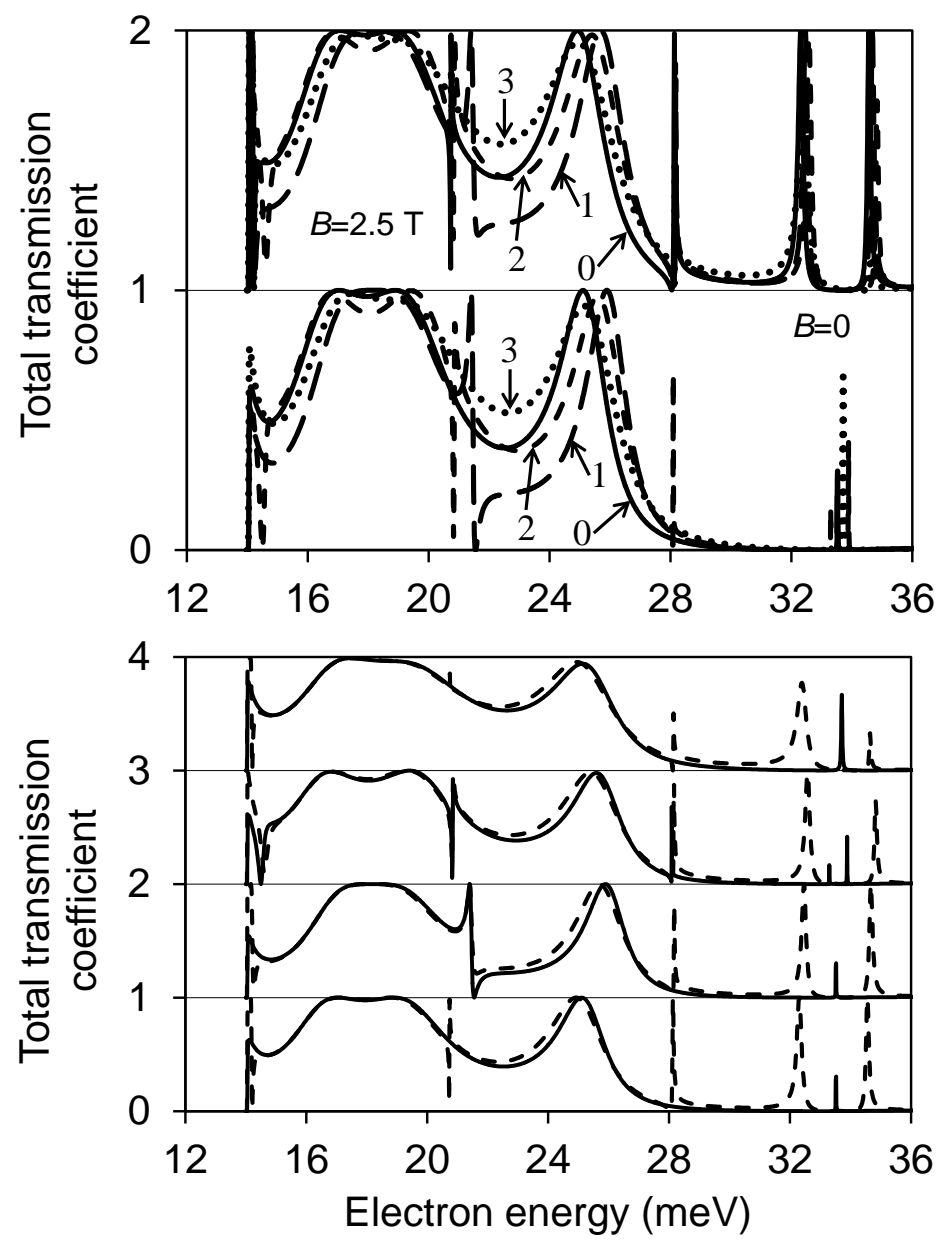

FIG. 8: Transmission plots for the rectangular ring showing the effects of the magnetic field and a single impurity. In the lower plot, the solid curves are results at zero field, and dotted lines are for $B=2.5 \mathrm{~T} \approx B_{p}$. From bottom to top, the results are for a ring with no impurity, followed by a single impurity of strength $V_{\mathrm{imp}}=t_{0}=0.14 \mathrm{eV}$ at locations 1,2 , and 3 . The upper plot shows the same results for each magnetic field. Results are labelled for a ring with no impurity (solid line, 0) and a single impurity at locations 1 (long dash), 2 (short dash), and 3 (dotted).

so that $t_{0}=0.14 \mathrm{eV}$. The locations we have chosen are marked in Fig. 7, and Fig. 8 shows the resulting effect on the transmission coefficient. 
A single impurity has relatively little effect on the transmission coefficient overall with the peaks showing little change in magnitude or energy. It is interesting, however, that the zero field results show almost identical Fano resonances to those at $2.5 \mathrm{~T}$ when the impurity is placed at site 1 or 2 , but this is not the case for site 3. Fig. 8 shows that an impurity at site 3 narrows the resonances when a magnetic field is present. We conclude from this and related studies that the Fano resonances are enhanced when the symmetry is broken in the $y$-direction, whilst making the device asymmetric in the $x$-direction can have the opposite effect. This is in agreement with the theory presented in the literature regarding the magnetic enhancement of Fano resonances ${ }^{13,16}$. The presence of well-defined resonances at zero field in Fig. 8 stand in stark contrast to those where there is no symmetry breaking in the $y$-direction, although technically the presence of resonances narrower than the energy resolution used, which is $1.25 \times 10^{-2} \mathrm{meV}$, cannot be ruled out.

The effect of Anderson-type disorder ${ }^{37}$ on the properties of the ring is now investigated, to mimic the effects of structural imperfections which may naturally arise during the manufacturing process as well as due to impurities. A potential, randomly chosen from a uniform distribution over the range $-V_{D}$ to $+V_{D}$ inclusive, is added to each grid point in the device region. Again, $V_{D}$ is measured in units of $t_{0}$, with $t_{0}=0.14 \mathrm{eV}$ for the parameters used. It turns out that even a weak disorder parameter of $V_{D}=0.01 t_{0}$ is sufficient to introduce just enough asymmetry between the arms to broaden previously-unresolved Fano resonances in the transmission coefficient to the extent that they can be resolved on an energy grid of spacing $1.25 \times 10^{-2} \mathrm{meV}$. The zero-field transmission results appear unchanged otherwise, although fluctuations can be seen in the Aharonov-Bohm oscillations of the transmission coefficient as a function of the magnetic field.

Higher disorder strengths have a more noticeable effect on the transmission coefficient and therefore the linear response transport coefficients. This is visible in Fig. 9 and Fig. 10, which show that the effect of the disorder is dependent on its strength and configuration. In general the conductance is reduced as the disorder makes it less probable that an electron will be able to pass through the device. The peaks also shift to lower energies. This means that the oscillations in the thermopower also move to lower energies although the maximum magnitudes of $S$ remain similar to those for the disorderless ring. Consequently, the maximum figure of merit can still be high with peak values of approximately 15, 10, and 

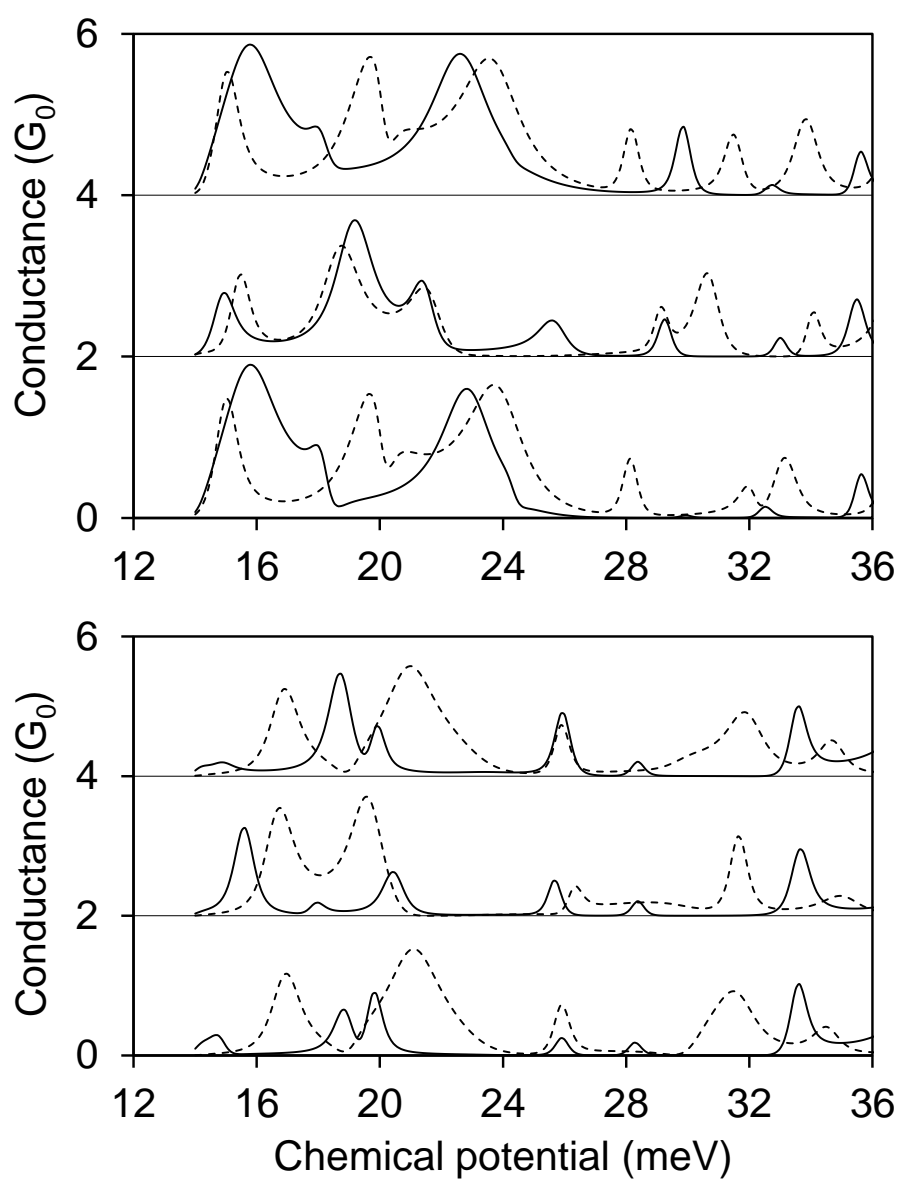

FIG. 9: Conductance for the disordered ring with disorder strengths $V_{D}=0.3 t_{0}$ (top) and $0.5 t_{0}$ (bottom), and $k_{B} T=0.125 \mathrm{meV}(T \approx 1.4 \mathrm{~K})$. Solid lines are for one random-disorder configuration and dotted lines are for a different configuration. Results for different values of the magnetic field are offset for clarity, with $B=0,1.25$, and $2.5 \mathrm{~T}$ from bottom to top in each plot.

5 for $V_{D}=0.5 t_{0}$ and $B=0,1.25$, and $2.5 \mathrm{~T}$ respectively, although the peaks as depicted in Fig. 5 move to lower energies. In other words, for $B=0$ and $B \approx B_{p}$, the maximum figure of merit is approximately half that of the ring without any disorder, whilst it remains almost unchanged for $B \approx 0.5 B_{p}$. However, all three values are still relatively large.

Finally, it is noted that the disorder leads to a breakdown of the Aharonov-Bohm effect. It has already been mentioned that even a small amount of disorder affects the AharonovBohm-oscillations by introducing pronounced fluctuations in the transmission coefficient. 

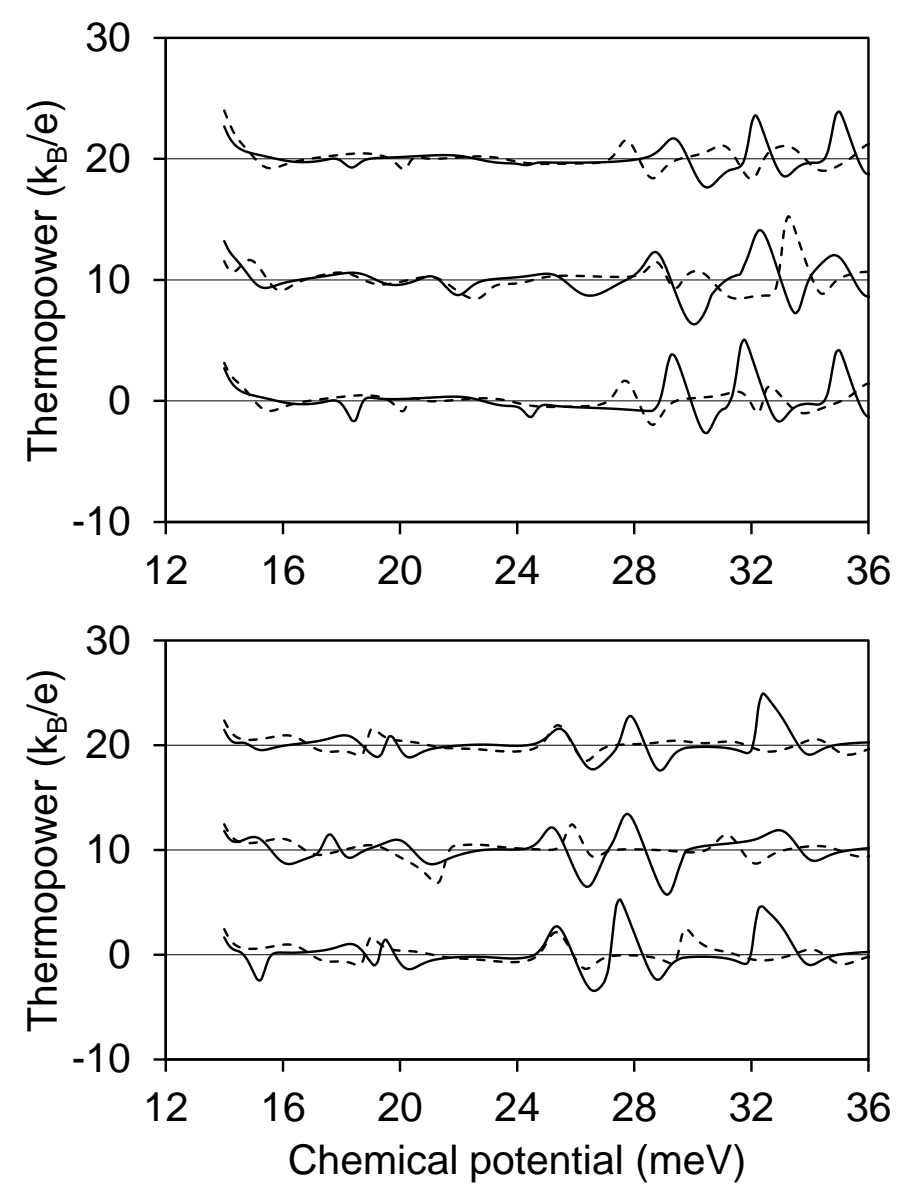

FIG. 10: Thermopower for the disordered ring with disorder strengths $V_{D}=0.3 t_{0}$ (top) and $0.5 t_{0}$ (bottom), and $k_{B} T=0.125 \mathrm{meV}(T \approx 1.4 \mathrm{~K})$. Solid lines are for one random-disorder configuration and dotted lines are for a different configuration. Results for different values of the magnetic field are offset for clarity, with $B=0,1.25$, and $2.5 \mathrm{~T}$ from bottom to top in each plot.

Fig. 9 shows that the change in the conductance with $B$ is greatly reduced when $V_{D}=0.3 t_{0}$, and increasing the disorder strength to $0.5 t_{0}$ reduces the effect still further. This is because the disorder on this scale limits the opportunities for constructive interference between paths which traverse the two arms of the ring. 


\section{CONCLUSIONS}

A theoretical investigation of the thermoelectric behavior of a rectangular AharonovBohm ring has been presented. The theory is based on a discretized Green's function approach which makes the inclusion of imperfections such as single-site impurities and Anderson disorder straightforward. The effect of the magnetic field in the leads, which is often neglected entirely in the literature, is accounted for by including a section of the leads as part of the actual device structure and only neglecting the magnetic field in the extremities of the leads. This simplifies the theory by still allowing many of the same expressions used at zero field to be used in the presence of a magnetic field (working in the Coulomb gauge). It is then also straightforward to obtain the contribution of each individual lead to the effective local density of states, allowing the physical behavior to be studied in greater depth.

The rectangular ring has a number of interesting characteristics. The transmission coefficient shows similar properties to that of single and double bends with peak-trough behavior at low energies and a stop-band at higher energies ${ }^{2}$. Both positive and negative peaks of large magnitude are seen in the thermopower which can be tuned by varying the magnetic field. In addition, fine resonances in the transmission coefficient in the stop-band region can result in a large thermoelectric figure of merit.

Under the application of a magnetic field, Aharonov-Bohm oscillations in the transmission coefficient are reproduced with a period in good agreement with theoretical predictions. We have also shown how the magnetic field results in the electrons coupling preferentially to either the upper or lower arm of the ring, depending on which lead they are incident from. The asymmetry introduced by this mechanism leads to a broadening of the Fano-like

resonances in the transmission coefficient, which we have further illustrated by considering the effects of having a single impurity placed at different locations in the ring.

The effect of Anderson disorder on the ring has also been examined. It is found that even a small level of disorder introduces pronounced fluctuations in the Aharonov-Bohm oscillations of the transmission coefficient as a function of magnetic field. Higher levels of disorder can cause the oscillations to break down completely, whilst reducing the electrical conductance and shifting the maxima in the thermopower to lower energies. The disorder 
reduces the thermoelectric efficiency but the thermoelectric figure of merit can still be large even in the presence of strong disorder. In conclusion, it appears that ring structures are equally as interesting as single and double bend structures for specialist thermoelectric applications at zero field, with the added feature that the presence of a magnetic field may be used for tuning purposes, at least in principle. Further work is needed to evaluate this potential in the context of specific applications and to make comparison with other possible technological approaches, for example the development of high performance micro-coolers based around semiconductor-superconductor junctions ${ }^{38}$.

\section{ACKNOWLEDGEMENTS}

A.J.P. acknowledges the support of Engineering and Physical Sciences Research Council (UK) for financial support through a Doctoral Training Grant. 


\section{REFERENCES}

${ }^{1}$ J. Zhou and R. Yang, "Ballistic thermoelectricity in double-bend nanowires," Appl. Phys. Lett. 98, 173107 (2011).

${ }^{2}$ A. J. Pye, D. A. Faux, and M. J. Kearney, "Coherent thermoelectric transport in single, double, and u-bend structures," J. Appl. Phys. 117, 064312 (2015).

${ }^{3}$ Y. Aharonov and D. Bohm, "Significance of electromagnetic potentials in the quantum theory," Phys. Rev. 115, 485 (1959).

${ }^{4}$ M. Büttiker, Y. Imry and M. Ya. Azbel, "Quantum oscillations in one-dimensional normal rings," Phys. Rev. A 30, 1982 (1984).

${ }^{5}$ R. A. Webb, S. Washburn, C. P. Umbach and R. B. Laibowitz, "Observation of $h / e$ Aharonov-Bohm oscillations in normal-metal rings," Phys. Rev. Lett. 54, 2696 (1985).

${ }^{6}$ R. A. Webb and S. Washburn, "Quantum interference fluctuations in disordered metals," Physics Today 41, 46 (1988).

${ }^{7}$ G. D. Guttman, E. Ben-Jacob and D. J. Bergman, "Thermopower of mesoscopic and disordered systems," Phys. Rev. B 51, 17758 (1995).

${ }^{8}$ Ya. M. Blanter, C. Bruder, R. Fazio and H. Schoeller, "Aharonov-Bohm oscillations of electrical and thermal properties of a quantum dot ring geometry," Czechoslovak J. Phys. 46, 2329 (1996).

${ }^{9}$ Ya. M. Blanter, C. Bruder, R. Fazio and H. Schoeller, "Aharonov-Bohm-type oscillations of thermopower in a quantum dot ring geometry," Phys. Rev. B 55, 4069 (1997).

${ }^{10} \mathrm{~T}-\mathrm{S}$. Kim and S. Hershfield, "Thermoelectric effects of an Aharonov-Bohm interferometer with an embedded quantum dot in the Kondo regime," Phys. Rev. B 67, 165313 (2003).

${ }^{11}$ J. Zheng, F. Chi, X-D. Lu and K-C. Zhang, "Thermoelectric effect in an Aharonov-Bohm ring with an embedded quantum dot," Nanoscale Research Lett. 7, 157 (2012).

${ }^{12}$ M. L. Ladrón de Guevara, F. Claro and P. A. Orellana, "Ghost Fano resonance in a double quantum dot molecule attached to leads," Phys. Rev. B 67, 195335 (2003).

${ }^{13}$ P. A. Orellana, M. L. Ladrón de Guevara and F. Claro, "Controlling Fano and Dicke effects via a magnetic flux in a two-site Anderson model," Phys. Rev. B 70, 233315 (2004).

${ }^{14}$ H-J. Xue, T-Q. Lü, H-C. Zhang, H-T. Yin, L. Cui and Z-L. He, "Thermospin effects in parallel coupled double quantum dots in the presence of the Rashba spin-orbit interaction and Zeeman splitting," Chin. Phys. B 21, 037201 (2012). 
${ }^{15}$ Y-S. Liu, D-B. Zhang, X-F. Yang and J-F. Feng, "The role of Coulomb interaction in thermoelectric effects of an Aharonov-Bohm interferometer," Nanotech. 22, 225201 (2011).

${ }^{16}$ G. Gómez-Silva, O. Ávalos-Ovando, M. L. de Guevara and P. A. Orellana, "Enhancement of thermoelectric efficiency and violation of the wiedemann-franz law due to fano effect," J. Appl. Phys. 111, 053704 (2012).

${ }^{17}$ O. Entin-Wohlman and A. Aharony, "Three-terminal thermoelectric transport under broken time-reversal symmetry," Phys. Rev. B 85, 085401 (2012).

${ }^{18}$ O. Entin-Wohlman, A. Aharony, and Y. Imry, "Mesoscopic aharonov-bohm interferometers: Decoherence and thermoelectric transport," arXiv preprint arXiv:1306.1813 (2013).

${ }^{19}$ V. Balachandran, G. Benenti, and G. Casati, "Efficiency of three-terminal thermoelectric transport under broken time-reversal symmetry," Phys. Rev. B 87, 165419 (2013).

${ }^{20}$ X. Lu, J. S. Wang, W. G. Morrel, X. Ni, C. Q. Wu and B. Li, "Thermoelectric effect in aharonovbohm structures," J. Phys. Condens. Matter 27, 035301 (2015).

${ }^{21} \mathrm{M}$. W. Wu, J. Zhou and Q. W. Shi, "Spin-dependent quantum transport in periodic magnetic modulations: Aharonov-Bohm ring structure as a spin filter," Appl. Phys. Lett. 85, $1012(2004)$.

${ }^{22}$ B. Wang, J. Zhou, R. Yang and B. Li, New. J. Phys. 16, 065018 (2014).

${ }^{23}$ R. Landauer, "Spatial variation of currents and fields due to localized scatterers in metallic conduction," IBM J. Res. Dev. 1, 223 (1957).

${ }^{24}$ M. Büttiker, "Four-terminal phase-coherent conductance," Phys. Rev. Lett. 57, 1761 (1986).

${ }^{25}$ M. Büttiker, Y. Imry, R. Landauer and S. Pinhas, "Generalized many-channel conductance formula with application to small rings," Phys. Rev. B 31, 6207 (1985).

${ }^{26}$ M. Büttiker, "Magnetoresistance of very pure simple metals," Phys. Rev. B 42, 3197 (1990).

${ }^{27}$ S. Datta, Electronic Transport in Mesoscopic Systems, Cambridge Studies in Semiconductor Physics and Microelectronic Engineering (Cambridge University Press, 1997).

${ }^{28} \mathrm{M}$. Governale and C. Ungarelli, "Gauge-invariant grid discretization of the Schrödinger equation," Phys. Rev. B 58, 7816 (1998).

${ }^{29}$ R. Peierls, "On the theory of diamagnetism of conduction electrons," in Selected Scientific Papers of Sir Rudolf Peierls, edited by R. Dalitz and R. Peierls (Imperial College Press, London, 1993) p. 97. 
${ }^{30}$ D. S. Fisher and P. A. Lee, "Relation between conductivity and transmission matrix," Phys. Rev. B 23, 6851 (1981).

${ }^{31}$ P. N. Butcher, "Thermal and electrical transport formalism for electronic mictostructures with many terminals," J. Phys.: Condens. Matter 2, 4869 (1990).

${ }^{32}$ H. van Houten, L. W. Molenkamp, C. W. J. Beenakker and C. T. Foxon, "Thermo-electric properties of quantum point contacts," Semicond. Sci. Technol. 7, B215 (1992).

${ }^{33}$ H. Goldsmid, Thermoelectric Refrigeration, The international cryogenics monograph series (Plenum Press, New York, 1964).

${ }^{34}$ A. Weisshaar, J. Lary, S. M. Goodnick and V. K. Tripathi, "Analysis of discontinuities in quantum waveguide structures," Appl. Phys. Lett. 55, 2114 (1989).

${ }^{35}$ M. Cutler and N. F. Mott, "Observation of Anderson localization in an electron gas," Phys. Rev. 181, 1336 (1969).

${ }^{36}$ P. Streda, "Quantised thermopower of a channel in the ballistic regime," J. Phys.: Condens. Matter 1, 1025 (1989).

${ }^{37} \mathrm{P}$. W. Anderson, "Absence of diffusion in certain random lattices," Phys. Rev. 109, 1492 (1958).

${ }^{38}$ D. Gunnarsson, J. S. Richardson-Bullock, M. J. Prest, H. Q. Nguyen, A. V. Timofeev, V. A. Shah, T. E. Whall, E. H. C. Parker, D. R. Leadley, M. Myronov and M. Prunnila, "Interfacial engineering of semiconductorsuperconductor junctions for high performance micro-coolers," Nature Sci. Rep. 5, 17398 (2015). 